\title{
Fabrication and Characterization of Sub-Micron Thin Film Intrinsic Josephson Junction Arrays
}

\author{
P. A. Warburton, A. R. Kuzhakhmetov, G. Burnell, M. G. Blamire, Y. Koval, A. Franz, P. Müller, and H. Schneidewind
}

\begin{abstract}
We have fabricated intrinsic Josephson junction arrays in thin films of $\mathrm{Tl}-\mathrm{Ba}-\mathrm{Ca}-\mathrm{Cu}-\mathrm{O}$. Such arrays are candidates for applications in the sub-millimeter waveband both by virtue of the large gap energy and due to the existence of inductive and capacitive coupling mechanisms between junctions in the array. Characterization of such junctions is complicated by the fact that the transport properties are dominated by Josephson fluxon flow (for junctions whose dimensions exceed the Josephson penetration depth) and premature switching to the voltage state at bias currents less than the critical current (for junctions whose dimensions are less than the Josephson penetration depth). Here we show that the magnetic-field dependence of the switching current is not Fraunhofer-like, although there is clear minimum corresponding to the point at which a single flux quantum is inserted between the cuprate double-planes. Nevertheless a Fraunhofer-like dependence can be obtained if the critical current is experimentally defined by specifying a low-voltage criterion.
\end{abstract}

Index Terms-Josephson junctions, superconductivity, thermal noise.

\section{INTRODUCTION}

$\mathbf{S}$ INCE the spacing of consecutive copper-oxide double planes in many cuprate superconductors is greater than the coherence length in the c-direction, such planes are Josephson coupled. Hence a cuprate superconductor which is structured in such a way that the current is forced to flow in the c-direction acts as a series array of "intrinsic" Josephson junctions [1]. Such junctions show promise for a wide variety of applications. For example, zero-crossing Shapiro steps at frequencies up to $2.5 \mathrm{THz}$ have been measured in single crystal $\mathrm{Bi}-\mathrm{Sr}-\mathrm{Ca}-\mathrm{Cu}-\mathrm{O}$ intrinsic junctions [2], suggesting that they are suitable for development as voltage standards and sub-mm-wave oscillators [3], [4]. A variety of thin-film-based device geometries has also been proposed, with a view to further exploiting intrinsic Josephson effects in device applications; for a review of such devices see [5].

The conventional way to confirm that Josephson coupling is the transport mechanism in any novel type of Josephson device is to measure the in-plane magnetic-field dependence of the critical current, $I_{c}(H)$. For a junction in which the phase difference

Manuscript received October 5, 2004. This work was supported in part by the U.K. Engineering and Physical Sciences Research Council.

P. A. Warburton and A. R. Kuzhakhmetov are with University College London, Department of Electrical and Electronic Engineering, Torrington Place, London, WC1E 7JE, U.K. (e-mail: p.warburton@ee.ucl.ac.uk).

G. Burnell and M. G. Blamire are with the University of Cambridge, Dept. of Materials Science, Cambridge, CB2 3QZ, U.K.

Y. Koval, A. Franz and P. Müller are with Universität Erlangen-Nürnberg, Physikalisches Institut III, D-91058, Erlangen, Germany.

H. Schneidewind is with IPHT-Jena, 07745 Jena, Germany.

Digital Object Identifier 10.1109/TASC.2005.849766 across the barrier is spatially uniform this dependence is of the form:

$$
I_{c}(H)=I_{c}(0)\left|\frac{\sin \left(\frac{\pi H}{H_{0}}\right)}{\frac{\pi H}{H_{0}}}\right|
$$

where $H_{0}$ is the field required to insert a flux quantum into a single junction. If, however, Josephson fluxons are present in the junction, the phase difference is highly spatially nonuniform, leading to significant deviations from the so-called Fraunhofer dependence of (1) [6]. In zero field such fluxons are present for junctions with linear dimension $w$ exceeding the Josephson penetration depth, $\lambda_{J}$. Small junctions, on the other hand, are susceptible to premature switching by thermal activation. Such premature switching occurs at a current, $I_{s w}$, which can be much less than $I_{c}$, rendering direct measurements of the Fraunhofer dependence impossible. As we will show below, for intrinsic Josephson junctions there exists no junction size $w$ in which there is neither penetration of Josephson fluxons nor premature switching at $4.2 \mathrm{~K}$. This suggests that measurements of $I_{s w}(H)$ should never be Fraunhofer-like at 4.2 K. Nevertheless we experimentally show that by defining a critical current $I_{c}$ at an arbitrary low voltage of order $\mu \mathrm{V}$, a Fraunhofer-like dependence can be observed.

\section{ANALYSIS}

Given that Josephson fluxons must be excluded, the maximum area of a junction in which one might expect to see the Fraunhofer dependence is of order $\lambda_{J}^{2}$. The maximum Josephson energy of such a junction is thus

$$
E_{J, \max }=\frac{\Phi_{0}}{4 \pi} j_{c} \lambda_{J}^{2}
$$

where $\Phi_{0}$ is the flux quantum and $j_{c}$ the critical current density. For intrinsic Josephson junctions, $\lambda_{J}$ is given by [7]

$$
\lambda_{J}=\left(\frac{\Phi_{0}}{2 \pi \mu_{0}} \frac{d}{\lambda_{a b}^{2} j_{c}}\right)^{\frac{1}{2}}
$$

where $\mu_{0}$ is the permeability of free space, $d \approx 3 \AA$ is the thickness of the cuprate double-planes, and $\lambda_{a b}$ is the in-plane penetration depth. By combining (2) and (3) we find that the maximum Josephson energy for an intrinsic junction which can be expected to show the Fraunhofer dependence is

$$
E_{J, \max }=\left(\frac{\Phi_{0}}{2 \pi \lambda_{a b}}\right)^{2} \frac{d}{\mu_{0}}
$$




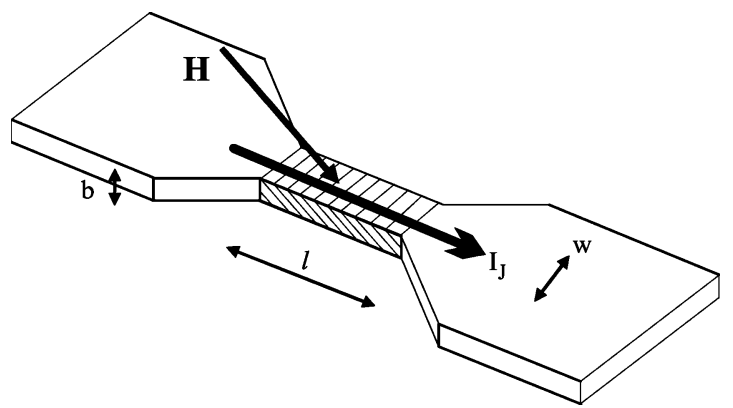

Fig. 1. Schematic (not to scale) of our device geometry. The slanting lines in the center of the structure indicate the orientation of the copper-oxide double planes. $\mathbf{H}$ shows the magnetic field vector and $\mathrm{I}_{\mathrm{J}}$ the transport current. The devices reported here are of length $l=3 \mu \mathrm{m}$, width $\mathrm{w}=0.5 \mu \mathrm{m}$ and film thickness $\mathrm{b}=240 \mathrm{~nm}$.

A Josephson junction switches to the voltage state by escape out of a potential well of height

$$
U=\frac{E_{J}}{2}(1-i)^{\frac{3}{2}}
$$

where $i=I / I_{c}$ is the normalized bias current [8]. Our model is based upon the simplifying assumption that thermal activation from this potential well occurs when $U=\eta k T$, where $\eta$ is a phenomenological factor of order 1 and $k$ is Boltzmann's constant. (This approximation is only applicable in the limit $E_{J}>$ $k T$. A full and more precise model applicable for $E_{J} \sim k T$ has been published in [9].) Under this assumption the normalized current at which switching to the voltage state occurs is given by

$$
i_{s w}=1-\left(\frac{2 \eta k T}{E_{J}}\right)^{\frac{2}{3}}
$$

By combining (4) and (6) we can now obtain the maximum value of the normalized switching current for a Josephson junction in which fluxons are excluded. By putting $\lambda_{a b}=200 \mathrm{~nm}$ and $\eta=1$ we find that $i_{s w, \max }=0.68$ at $T=4.2 \mathrm{~K}$-i.e. in junctions in which we can expect the critical current to follow the Fraunhofer dependence, the apparent critical current, $I_{s w}$, is at most $68 \%$ of the actual critical current, $I_{c}$, at the usual measurement temperature. Conversely, if we pose the question "at what temperature should measurements be made so that the apparent critical current is within 5\% is the actual critical current?" we find an upper limit for this temperature to be $260 \mathrm{mK}$.

\section{EXPERIMENTAL}

Our device geometry which makes use of mis-aligned $\mathrm{Tl}_{2} \mathrm{Ba}_{2} \mathrm{CaCu}_{2} \mathrm{O}_{8}$ films is shown in Fig. 1. The substrate is vicinal lanthanum aluminate with its surface cut at an angle of $20^{\circ}$ to the $\left(\begin{array}{lll}0 & 0 & 1\end{array}\right)$ axis. The films, of thickness $240 \mathrm{~nm}$ are grown by sputtering an amorphous $\mathrm{Ba}-\mathrm{Ca}-\mathrm{Cu}-\mathrm{O}$ precursor and ex situ annealing in a thallous atmosphere at around $800^{\circ} \mathrm{C}$ [10]. During the annealing process the TBCCO film recrystallizes epitaxially on the vicinal substrate with the result that the copper-oxide double planes are mis-aligned by $20^{\circ}$ with respect to the substrate surface. This epitaxy is confirmed both by four-circle $\mathrm{x}$-ray diffraction and cross-sectional transmission electron micrography [11]. Micron-scale features are patterned

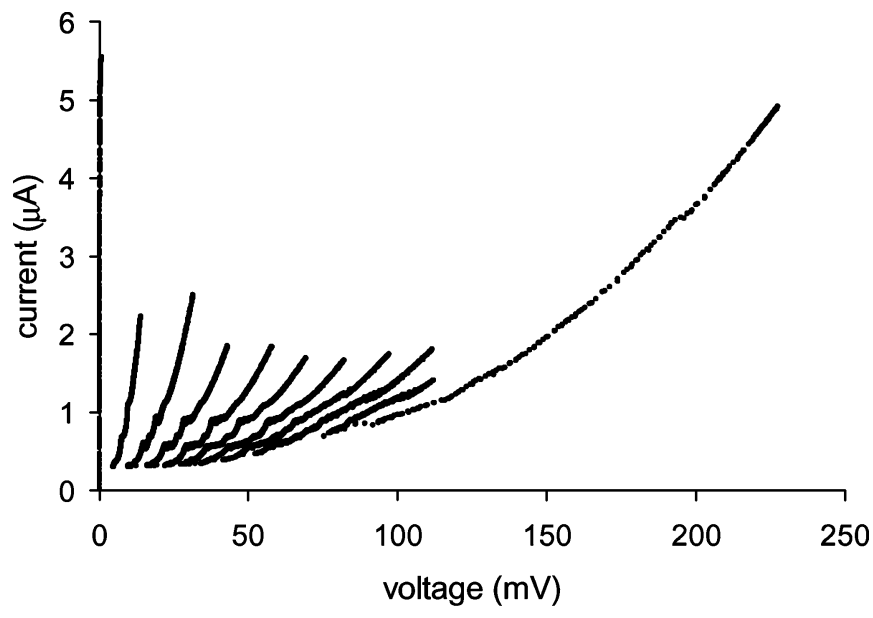

(a)

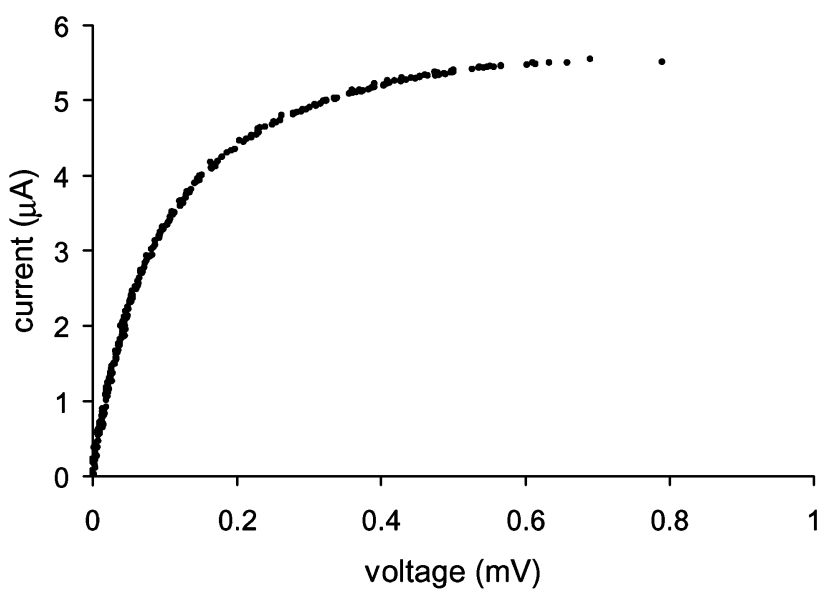

(b)

Fig. 2. (a) Part of the current-voltage characteristics of an intrinsic Josephson junction array in zero magnetic field at $T=4.2 \mathrm{~K}$. Only ten of the quasiparticle branches are shown here. (b) Enlargement of the low-voltage region of (a).

using argon-ion milling and sub-micron features using a 30 $\mathrm{keV}$ gallium focussed ion-beam.

Transport measurements in zero magnetic field are performed by immersing the sample into a liquid helium storage vessel. Measurements in a magnetic field of up to $3 \mathrm{~T}$ are performed in an Oxford Instruments helium cryostat. The sample may be rotated with respect to the field with angular resolution better than $0.1^{\circ}$. This high precision allows us to avoid inserting pancake vortices, for which it is essential that the misalignment between the applied field vector and the copper-oxide planes be less than $\theta_{\text {mis, } \text { max }}=H_{c 1 \| c} / H$, where $H_{c 1 \| c}$ is the lower critical field of $\mathrm{Tl}_{2} \mathrm{Ba}_{2} \mathrm{CaCu}_{2} \mathrm{O}_{8}$ in the c-direction. For our maximum applied field of $3 \mathrm{~T}$ this implies that the misalignment must be smaller than $\sim 0.4^{\circ}$. Details of the alignment procedure can be found elsewhere [12].

\section{RESULTS}

\section{A. Current-Voltage Characteristics}

The current-voltage characteristics of a device of width $w=0.5 \mu \mathrm{m}$ are shown in Fig. 2. The characteristics are multi-branched (in common with all intrinsic Josephson devices), each branch corresponding to an additional junction 


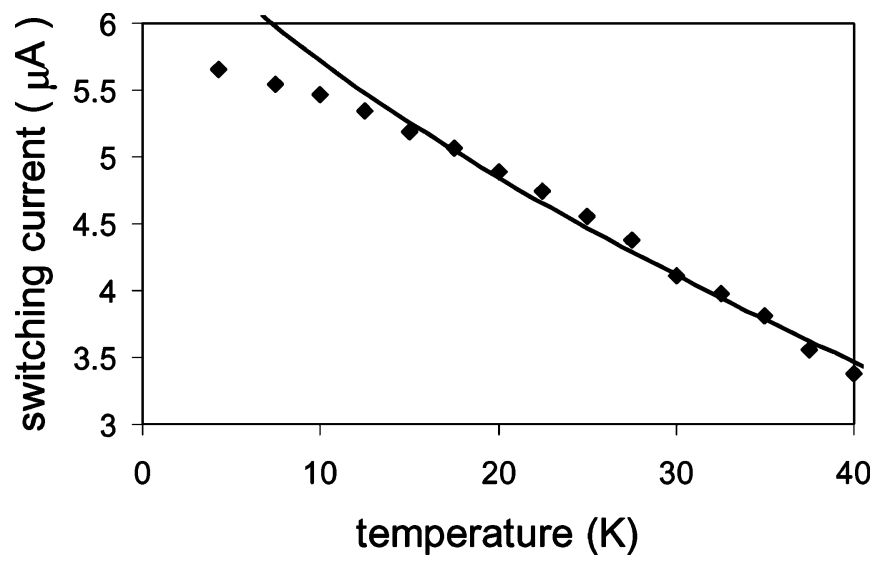

Fig. 3. Temperature dependence of the switching current in zero field. The points are the experimental data and the line is a fit using the thermal activation model of equation (6). The fitting parameter is $\eta=0.8$ (see text for details).

entering the voltage state. The reason for higher order branches switching at lower apparent critical current values is not understood. The supercurrent branch is not at zero voltage, the dissipation here being due to thermally-activated phase diffusion [12], [13]. The current at which the jump from the supercurrent branch occurs (equal to $5.5 \mu \mathrm{A}$ here) is therefore not the Josephson critical current. We have previously shown by studying the temperature dependence of the supercurrent branch in this device that the zero-field low-temperature critical current is $7.2 \mu \mathrm{A}$ [13], corresponding to a Josephson energy of $170 k \mathrm{~K}$. This critical current, in common with BCS superconductors, is approximately constant for temperatures less than half the superconducting transition temperature.

\section{B. Temperature Dependence of the Switching Current}

The temperature dependence of the switching current is shown in Fig. 3, where we restrict ourselves to $T<40 \mathrm{~K}$, in which regime the critical current is essentially constant. We now use our simplified thermal activation model (6) to account for the temperature dependence of $I_{s w}$. This dependence is shown in Fig. 3, with $E_{J} / k=170 \mathrm{~K}$ as described in the previous section. The only fitting parameter is the phenomenological term $\eta$, which is 0.8 here. We conclude that, although the model is clearly oversimplified, it is adequate for giving order of magnitude estimates for the degree to which measurements of the switching current underestimate the critical current in small intrinsic Josephson junctions with $E_{J}>k T$.

\section{Magnetic-Field Dependence of the Switching Current}

The dependence of the switching current, normalized to its zero-field value, upon an in-plane aligned magnetic field is shown in Fig. 4. There is a clear minimum at $H=2.5 \mathrm{~T}$. The field required to insert a single flux quantum per intrinsic junction is given by [7]

$$
H_{0}=\frac{\Phi_{0}}{w(s+t)}
$$

where $s$ is the separation of the cuprate double-planes. Inserting the appropriate values for this device we find $H_{0}=2.37 \mathrm{~T}$, in reasonable agreement with the measured minimum, confirming

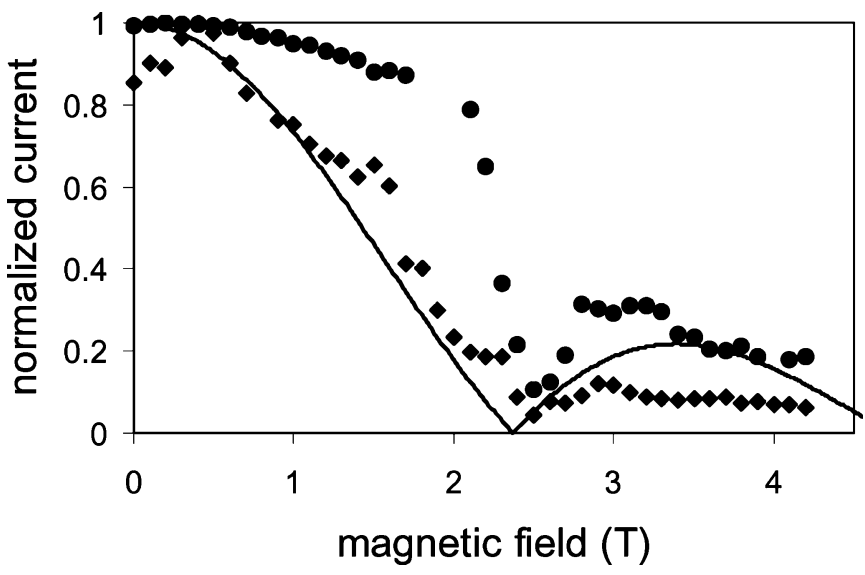

Fig. 4. Dependence of the switching current (circles) and critical current (diamonds) upon an in-plane aligned magnetic field at $T=4.2 \mathrm{~K}$, in both cases normalized to their respective zero-field values. The critical current is defined at a voltage criterion of $5 \mu \mathrm{V}$. The line shows the Fraunhofer dependence (equation (1)) with $H_{0}=2.37 \mathrm{~T}$ as the fitting parameter.

that the Josephson phenomena we observe are indeed intrinsic to the cuprate double-planes.

Nevertheless it is clear that the switching current dependence does not follow the Fraunhofer dependence, which is to be expected given that thermal fluctuations play a strong role in the switching process. We therefore also plot in Fig. 4 the magnetic-field dependence of the critical current defined at an arbitrary voltage criterion of $5 \mu \mathrm{V}$. Here the data are normalized to the zero-field value which is $190 \mathrm{nA}$. The fit to the Fraunhofer dependence (1) suggests that this low-voltage definition of the critical current is a more accurate measure of the true critical current than is the switching current. Care must therefore be taken in interpreting the critical-current field-dependence data for all small intrinsic Josephson junctions at $4.2 \mathrm{~K}$.

\section{Conclusions}

We have shown that due to premature switching it is not possible to directly measure the Fraunhofer dependence of the critical current in intrinsic Josephson junctions at $4.2 \mathrm{~K}$. We estimate an upper bound on the temperature for such measurements to be successful to be $260 \mathrm{mK}$. We have further developed a simplified model of thermally-activated premature switching of Josephson junctions with $E_{J}>k T$. This model allows us to estimate the magnitude by which measurements of the switching current underestimate the critical current.

\section{REFERENCES}

[1] R. Kleiner, F. Steinmeyer, G. Kunkel, and P. Mueller, "Intrinsic Josephson effects in Bi2sr2cacu2o8 single-crystals," Phys. Rev. Lett., vol. 68, p. 2394, 1992.

[2] H. B. Wang, P. H. Wu, and T. Yamashita, "Terahertz responses of intrinsic Josephson junctions in high Tc superconductors," Phys. Rev. Lett., vol. 87, p. $107002,2001$.

[3] M. Bae, H. Lee, J. Kim, and K. Kim, "Microwave distribution in stacked $\mathrm{Bi} 2 \mathrm{Sr} 2 \mathrm{CaCu} 2 \mathrm{O} 8+\mathrm{x}$ intrinsic Josephson junctions in a transmission-line geometry," Appl. Phys. Lett., vol. 83, p. 2187, 2003.

[4] H. Wang, J. Chen, P. Wu, T. Yamashita, D. Vasyukov, and P. Muller, "Intrinsic Josephson junctions: integrated circuits and possible applications," Supercond. Sci. Technol., vol. 16, p. 1375, 2003.

[5] A. A. Yurgens, "Intrinsic Josephson junctions: recent developments," Supercond. Sci. Technol., vol. 13, p. R85, 2000. 
[6] A. Barone and G. Paterno, Physics and Applications of the Josephson Effect. New York: Wiley, 1988.

[7] R. Kleiner and P. Müller, "Intrinsic Josephson effects in high-Tc superconductors," Phys. Rev. B, vol. 49, p. 1327, 1994.

[8] M. Tinkham, Introduction to Superconductivity, 2nd ed. New York: McGraw-Hill, 1996.

[9] A. Franz, Y. Koval, D. Vasyukov, P. Muller, H. Schneidewind, D. A. Ryndyk, J. Keller, and C. Helm, "Thermal fluctuations in ultrasmall intrinsic Josephson junctions," Phys. Rev. B, vol. 69, p. 014 506, 2004.

[10] H. Schneidewind, M. Manzel, G. Bruchlos, and K. Kirsch, "Tl$\mathrm{BaCaCuO}-(2212)$ thin films on lanthanum aluminate and sapphire substrates for microwave filters," Supercond. Sci. Technol., vol. 14, p. 200, 2001.
[11] O. S. Chana, A. R. Kuzhakhmetov, D. M. C. Hyland, C. J. Eastell, D. Dew-Hughes, C. R. M. Grovenor, Y. Koval, M. Moessle, R. Kleiner, P. Müller, and P. A. Warburton, "Fabrication and measurement of intrinsic Josephson junctions in misaligned films of T12Ba2CaCu2O8.," IEEE Trans. Appl. Supercond., vol. 11, p. 2711, 2001.

[12] P. Warburton, A. Kuzhakhmetov, O. Chana, G. Burnell, M. Blamire, H. Schneidewind, Y. Koval, A. Franz, P. Muller, D. Hyland, D. DewHughes, H. Wu, and C. Grovenor, "Josephson fluxon flow and phase diffusion in thin-film intrinsic Josephson junctions," J. Appl. Phys., vol. 95, p. 4941, 2004

[13] P. A. Warburton, A. R. Kuzhakhmetov, G. Burnell, M. G. Blamire, and H. Schneidewind, "Decoupling of a small current-biased Josephson junction from its environment," Phys. Rev. B, vol. 67, p. 184 513, 2003. 